\title{
Walker-Warburg syndrome: rare congenital muscular dystrophy associated with brain and eye abnormalities
}

\author{
CY Lee *
}

Hong Kong Med J 2014;20:556.e4-5

DOI: $10.12809 / \mathrm{hkmj} 134137$

\section{Case report}

A 7-month-old boy was found to have developmental delay, abnormal muscle tone, and abnormal eye movement in December 2012. Physical examination of the eyes revealed wandering gaze with convergent squint. Ophthalmology was consulted and bilateral retrolental masses were suspected. Blood tests revealed elevated serum creatine kinase level.

Computed tomography of orbits showed bilateral hyperdense retrolental tubular opacities with small retinal haemorrhage on the right (Fig 1). Computed tomography of brain also showed communicating hydrocephalus. Magnetic resonance imaging of orbits showed deformed bilateral globes, abnormal T1-weighted and T2-weighted hypo-toiso-intense contrast-enhancing triangular bands with base near the optic disc and apex at the posterior surface of lens, compatible with bilateral persistent hyperplastic primary vitreous. T1- and T2-weighted hyperintensity at right vitreous body was compatible with previous haemorrhage.

Magnetic resonance imaging of the brain showed pachygyria, hydrocephalus, absent septum pellucidum, and hypoplasia of corpus callosum forming type II lissencephaly (Fig 2). Mega cisterna magna, hypoplastic pons, and cerebellar vermis were compatible with posterior cranial fossa malformation (Fig 3). Band-like structures in the bilateral

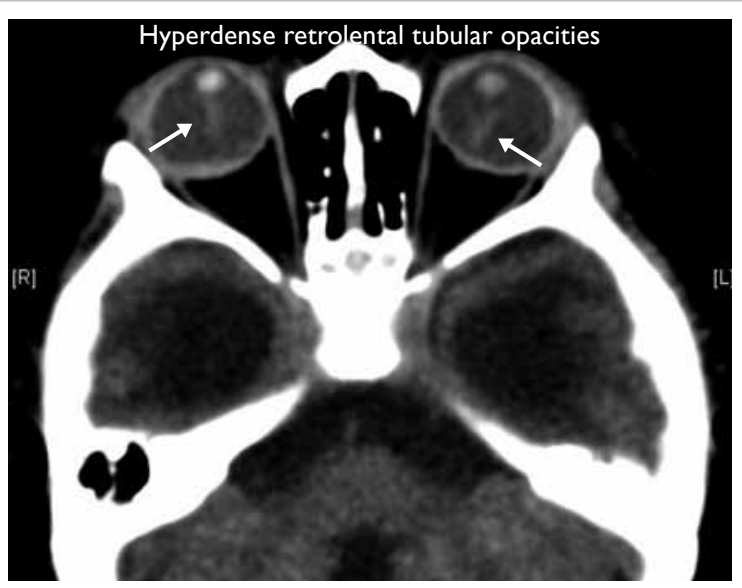

FIG I. Computed tomography of orbits shows bilateral, hyperdense retrolental tubular opacities (arrows), later confirmed to be bilateral, persistent hyperplastic primary vitreous on magnetic resonance imaging periventricular regions with signal changes similar to grey matter were suggestive of band heterotopic grey matter.

Radiological findings of type II lissencephaly, posterior fossa malformation and retinal anomaly, together with clinical findings of developmental delay, abnormal muscle tone, and elevated serum creatine kinase level were compatible with diagnosis of Walker-Warburg syndrome.

\section{Discussion}

Congenital muscular dystrophy (CMD) comprises a heterogeneous group of disorders. Walker-Warburg syndrome is one phenotype of CMD known to occur due to dystroglycanopathy, ${ }^{1}$ which is an autosomal recessive condition. The overall incidence is unknown but a survey in Northeastern Italy has reported an incidence rate of 1.2 per 100000 live births. ${ }^{2}$

Walker-Warburg syndrome affects the brain, eye, and muscles with characteristic malformation. Diagnostic criteria for WalkerWarburg syndrome include type II lissencephaly, cerebellar malformation, retinal malformation,

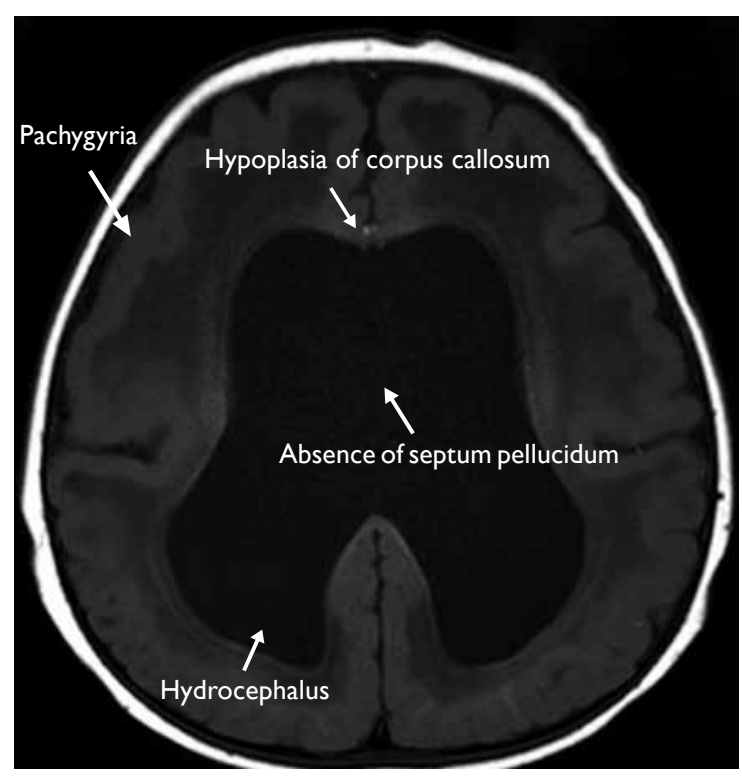

FIG 2. TI-weighted axial magnetic resonance imaging of brain shows pachygyria, hydrocephalus, absent septum pellucidum, and hypoplasia of corpus callosum forming type II lissencephaly (arrows) 


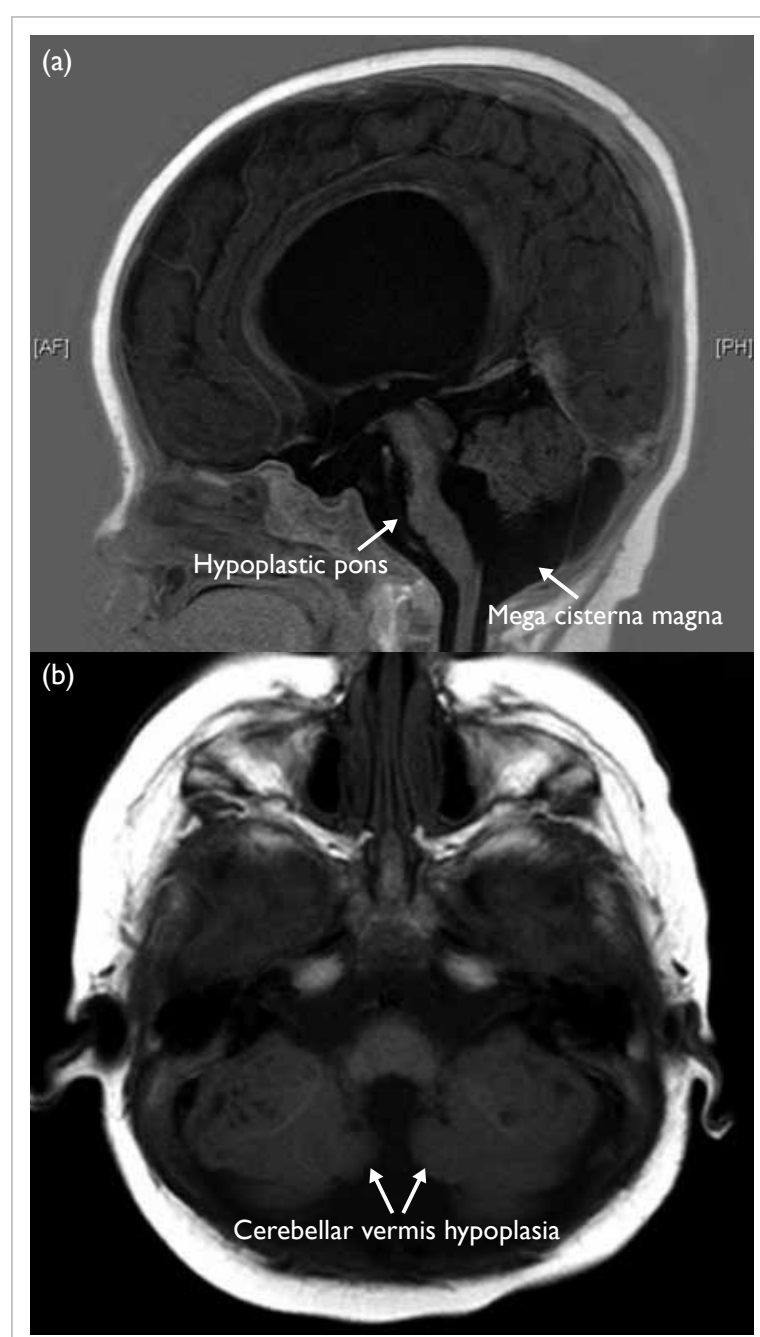

FIG 3. (a) TI-weighted sagittal and (b) axial magnetic resonance imaging of brain show mega cisterna magna, hypoplastic pons, and cerebellar vermis compatible with posterior cranial fossa malformation (arrows)

and CMD. ${ }^{3}$ Common associated anomalies include anterior chamber malformation of the eye and hydrocephalus. The less commonly observed anomalies include Dandy-Walker malformation, cleft lip and palate, congenital macrocephaly or microcephaly, posterior encephalocoele, ocular colobomas, congenital cataracts, and genital abnormalities. Neuroimaging findings other than lissencephaly include band heterotopia, cerebellar vermian hypoplasia, dysgenesis of corpus callosum, abnormal white matter changes, hypoplastic cerebral peduncles, intraventricular haemorrhage, cerebellar polymicrogyria, collicular fusion, and fusion of occipital poles. ${ }^{4}$ Laboratory investigations usually show elevated serum creatine kinase level, myopathic/dystrophic muscle pathology, and altered alpha-dystroglycan. ${ }^{2}$

Differentiation of Walker-Warburg syndrome from other dystroglycanopathies, for example, muscle-eye-brain disease or Fukuyama CMD, depends on the severity of clinical presentation including motor function and intellectual disability, and involvement of the central nervous system and eye. ${ }^{1}$ Walker-Warburg syndrome is believed to be the most severe form of dystroglycanopathy with most children dying before the age of 3 years. ${ }^{2}$ No specific treatment is available for this syndrome. Management is mainly supportive and preventive.

CY Lee *, FRCR, MB, ChB

Department of Radiology, Tuen Mun Hospital, Tuen Mun, Hong Kong

* Corresponding author: prodigycat@gmail.com

\section{References}

1. Sparks S, Quijano-Roy S, Harper A, et al. Congenital Muscular Dystrophy Overview. 2001 Jan 22 [Updated 2012 Aug 23]. In: Pagon RA, Adam MP, Ardinger HH, et al, editors. GeneReviews ${ }^{\ominus}$ [Internet]. Seattle (WA): University of Washington, Seattle; 1993-2014. Available from: http:// www.ncbi.nlm.nih.gov/books/NBK1291/. Accessed Sep 2013.

2. Vajsar J, Schachter H. Walker-Warburg syndrome. Orphanet J Rare Dis 2006;1:29.

3. Dobyns WB, Pagon RA, Armstrong D, et al. Diagnostic criteria for Walker-Warburg syndrome. Am J Med Genet 1989;32:195-210.

4. Zaleski CG, Abdenour GE. Pediatric case of the day. Walker-Warburg syndrome (cerebro-ocular dysplasiamuscular dystrophy). Radiographics 1997;17:1319-23. 